\title{
Constraints on dust composition in the Magellanic Clouds from gas-phase zinc, silicon, chromium, and iron abundances
}

\author{
Kirill Tchernyshyov ${ }^{* a}$, Jonathan P. Seale ${ }^{a}$, Margaret Meixner ${ }^{a, b}$, Andrew J. Fox ${ }^{b}$, \\ Scott D. Friedman ${ }^{b}$, Kenneth Sembach $^{b}$, Eli Dwek ${ }^{c}$, Frédéric Galliano ${ }^{d}$ \\ ${ }^{a}$ Dept. of Physics and Astronomy, Johns Hopkins University, 3400 N. Charles St., Baltimore, \\ MD 21218 \\ ${ }^{b}$ Space Telescope Science Institute, 3700 San Martin Dr., Baltimore, MD 21218, USA \\ ${ }^{c}$ NASA/GSFC, Code 665, Observational Cosmology Lab, Greenbelt, MD \\ ${ }^{d}$ Laboratoire AIM, CEA / Saclay, L'Orme des Merisiers, 91191, Gif-sur-Yvette, France \\ E-mail: ktchernyshyov@pha. jhu.edu, seale@pha. jhu.edu, \\ meixner@stsci.edu, afoxestsci.edu, friedmanestsci.edu, \\ sembach@stsci.edu, eli.dwek@nasa.gov, frederic.galliano@cea.fr
}

\begin{abstract}
We present near-ultraviolet Hubble Space Telescope Cosmic Origins Spectrograph (HST-COS) spectra towards sixteen stars in the Large and Small Magellanic Clouds. We use these spectra, as well as 109 archival Far Ultraviolet Spectroscopic Explorer (FUSE) spectra to measure the gasphase abundances of phosphorus, zinc, silicon, chromium, and iron in the interstellar medium of the Large and Small Magellanic Clouds. We find that the gas-phase to total interstellar medium abundance ratios of these elements are approximately the same as in the Milky Way for phosphorus and zinc and higher than in the Milky Way for silicon, chromium, and iron. This effect is greater in the Small Magellanic Cloud than in the Large Magellanic Cloud. We use these relative abundances to estimate the gas-to-dust mass ratios of the Large and Small Magellanic Clouds.
\end{abstract}

The Life Cycle of Dust in the Universe: Observations, Theory, and Laboratory Experiments - LCDU 2013, 18-22 November 2013

Taipei, Taiwan

\footnotetext{
* Speaker.
} 


\section{Introduction}

Interstellar dust plays an important role in star formation and the chemistry of the interstellar medium (ISM). Our current understanding of its chemical composition (e.g. [1]) is mostly based on its bulk optical properties, its emission lines, and comparisons of observed abundances of various elements in interstellar gas clouds with their total ISM abundances. The difference between the observed and expected abundance of an element in interstellar gas is the maximum possible abundance of that element in interstellar dust. Most of these studies have focused on either the Milky Way (MW) [2,3] or damped Lyman- $\alpha$ systems (DLAs) [4]. These two sets of observations represent environments with very different metallicities. There have not been as many studies of the gas-phase abundances of intermediate-metallicity environments.

The Large and Small Magellanic Clouds (LMC and SMC) are a pair of nearby irregular dwarf galaxies with metallicities of approximately $1 / 2$ and $1 / 5$ solar, respectively. Studies of the gasto-dust mass ratio (GDR) in the local universe have found that it tends to evolve linearly with metallicity until approximately the SMC value, at which point it begins to decrease super-linearly [5]. Ultraviolet extinction curves in the SMC and LMC are known to differ substantially from those in most of the MW [6,7]. The amount of thermal emission at submillimeter wavelengths by dust at metallicities close to those of the LMC and SMC is greater than is expected from studies of the emission properties of dust in the MW [8]. If there are differences in the chemical composition of dust at low metallicity relative to that of dust in the MW, the LMC and SMC are a convenient place to observe them.

In this study, we measure gas-phase abundances in the ISM of the LMC and SMC using ultraviolet absorption spectroscopy. We observe stars in the LMC and SMC and measure absorption lines of singly ionized phosphorus (P II), zinc (Zn II), silicon (Si II), chromium (Cr II), and iron (Fe II). The ionization potentials needed to doubly ionize these elements are low enough that only trace amounts of the singly ionized states would be present in the photospheres of the O-type background stars. This means that all of the observed absorption is due to the intervening ISM. The amount of absorption by a species $\mathrm{X}$ can be used to infer that species' column density $N(\mathrm{X})$.

The neutral atomic (H I) and molecular $\left(\mathrm{H}_{2}\right)$ hydrogen column densities of the ISM towards our background sources is typical of superpositions of diffuse atomic and diffuse molecular clouds [9]. Studies of gas-phase abundances in the MW indicate that substantial amounts of dust growth or destruction must occur in the diffuse atomic and molecular medium [3]. The GDRs of the LMC and SMC have been shown to be greater (i.e. more gas mass relative to dust mass) than in the MW using dust emission measurements ([10], [11]). The goals of this study include determining if dust growth or destruction is as prevalent in the diffuse atomic and molecular gas of the LMC and SMC as it is in the MW and estimating elemental abundances and, with some assumptions, GDRs in the LMC and SMC.

In Section 2, we describe new and archival observations used in this work. In Section 3, we describe our data analysis procedure and the inaccuracies it could potentially introduce. In Section 4, we discuss our results on depletion, the GDR, and dust composition in the LMC and SMC. 

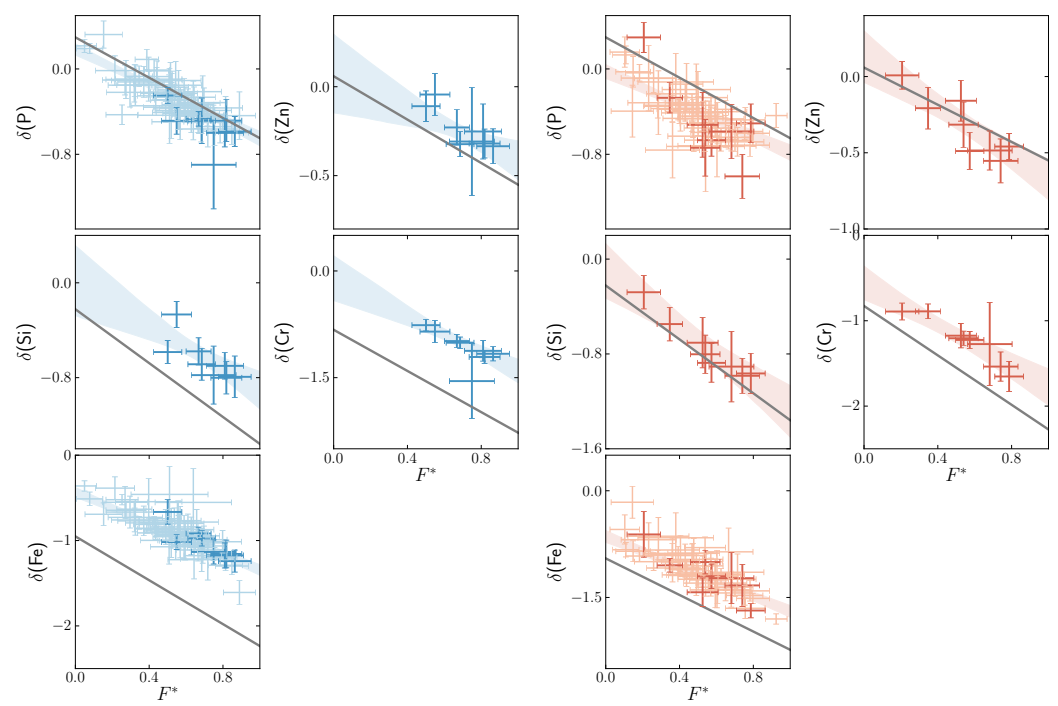

Figure 1: Fits of the Jenkins parametrization to elemental depletions in the SMC ISM. Dark and light points denote sightlines with and without COS observations; red and blue points represent sightlines in the LMC and SMC. Shaded regions show $68 \%$ credible intervals about the median fit. The gray line is the MW best fit from Jenkins [3]. $\delta(\mathrm{X})$ is the ratio of the observed abundance of an element $\mathrm{X}$ to its total ISM abundance. $F^{*}$ is an overall depletion factor which summarizes the depletions of all elements along a given sightline.

\section{Data}

Our observations include sixteen new near ultraviolet (NUV) spectra and 109 archival far ultraviolet (FUV) spectra. The NUV spectra were taken using the Cosmic Origins Spectrograph (COS) [12] as part of Hubble Space Telescope program ID 13004 (PI: Meixner, M.). The FUV spectra are from the Far Ultraviolet Spectroscopic Explorer (FUSE) Magellanic Clouds Legacy Project archive [13]. Both sets of spectra have a velocity resolution of approximately $15 \mathrm{~km} \mathrm{sec}^{-1}$ and typical signal-to-noise ratios of 10-50.

\section{Analysis}

In this section, we describe our techniques for inferring ion column densities from spectra, computing gas-phase abundances and depletions from column densities, and estimating GDRs.

We infer the column densities of the ions P II, Zn II, Si II, Cr II, and Fe II from absorption in our spectra using a variation of Voigt profile fitting. We define a likelihood function based on the assumption that an observed spectrum should be a draw from a multivariate normal distribution with a diagonal covariance matrix with variances equal to the estimated uncertainties and means equal to the model spectrum and draw samples from it using reversible jump Monte Carlo (RJMC) [14]. RJMC is a technique for drawing samples from probability distributions over varying numbers of parameters. We need such a technique because we allow the number of velocity components along the line of sight to vary, which means the number of velocity component centers, widths, and associated column densities is not constant. 


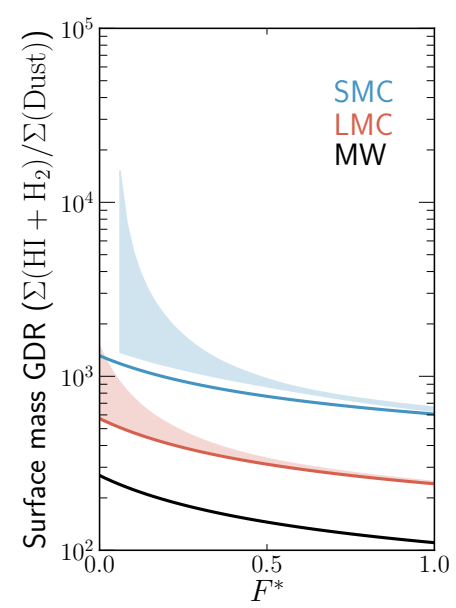

Figure 2: Surface mass GDR plotted against the generalized depletion parameter $F^{*}$. The MW, LMC, and SMC are denoted by black, red, and blue, respectively. Shaded regions show predictions based on observed LMC and SMC Si and Fe gas-phase abundances. Solid lines show scalings of the MW GDR by the LMC, and SMC metallicities, which are assumed to be $1 / 2$ and $1 / 5$ relative to solar; this corresponds to assuming MW, LMC, and SMC depletion patterns are identical. The Fe and Si-based GDR estimate is consistent with the scaled MW value in the LMC and greater than the scaled MW value in the SMC.

We assume all the metals we observe are coincident with $\mathrm{H} \mathrm{I}$ and $\mathrm{H}_{2}$ only and that ionization corrections are minimal. Our gas-phase abundances are equal to our inferred metal column densities divided by the sum of the $\mathrm{H}$ I and twice the $\mathrm{H}_{2}$ column densities from Welty, Xue, and Wong [15]. We generated a number of theoretical models of possible diffuse neutral medium cloud ionization structures using the Cloudy ([16], v13.02) photoionization code to test this assumption. P II and Si II may overestimate the $\mathrm{P}$ and $\mathrm{Si}$ abundances by a factor of up to three along sightlines with hydrogen column densities below $10^{20.5} \mathrm{~cm}^{-2}$. Zn II may underestimate the $\mathrm{Zn}$ abundances by a factor of up to 1.5 along sightlines with hydrogen column densities above $10^{21.5} \mathrm{~cm}^{-2}$. All of the sightlines along which we observe Si II have hydrogen column densities greater than $10^{20.5} \mathrm{~cm}^{-2}$. We may be underestimating the amount of zinc along six of our sightlines, which have hydrogen column densities greater than $10^{21.5} \mathrm{~cm}^{-2}$. This would translate into an overestimate of the amount of zinc missing from the gas-phase.

We divide our gas-phase abundances by assumed total ISM abundances to get depletions $\delta(\mathrm{X})$ for each element X. Our total ISM abundances are combinations of scaled solar values [17] and photospheric abundance measurements in young LMC and SMC stars (e.g. [18]). We use the Jenkins depletion parametrization [3] to combine the depletions of different elements into an overall depletion factor $F_{i}^{*}$ for each sightline. This allows us to predict Zn II, Si II, and Cr II depletions along sightlines which only have FUSE spectra. The Jenkins depletion parametrization is based on the observation that in the MW, elements deplete together. For example, if $\mathrm{Fe}$ is more depleted along sightline A than sightline $\mathrm{B}$, then $\mathrm{Si}$ is also more depleted along sightline A than sightline $\mathrm{B}$. The depletion $\delta(\mathrm{X})_{i}$ of an element $\mathrm{X}$ along a sightline $i$ is given by

$$
\delta(\mathrm{X})_{i}=\delta(\mathrm{X})_{0}+F_{i}^{*} A_{\mathrm{X}},
$$

where $\delta(\mathrm{X})_{0}$ and $A_{\mathrm{X}}$ are fixed across different sightlines and $F_{i}^{*}$ is fixed across different elements. 
Low and high values of $F^{*}$ correspond to sightlines with small and large depletions, respectively, of all elements. $A_{\mathrm{X}}$ and $\delta(\mathrm{X})_{0}$ are the slope and intercept of the linear relation between the overall depletion $F^{*}$ and depletion of element $\mathrm{X} \delta(\mathrm{X})$. Figure 1 shows fits of this parametrization to our depletion measurements.

We also use our measured gas-phase and assumed total ISM abundances to estimate the GDR. The amount of $\mathrm{Si}$ and $\mathrm{Fe}$ in dust has to be less than or equal to the amount of $\mathrm{Si}$ and $\mathrm{Fe}$ missing from the gas. If we assume that all of element $\mathrm{X}$ is in dust or gas, then the solid-phase element abundance $\varepsilon_{\text {dust }}(\mathrm{X})$ is the difference between the total ISM and measured gas-phase abundances. The dust-to-gas ratio (DGR), which is the inverse of the GDR, can be written as

$$
\begin{aligned}
\frac{M(\text { Dust })}{M(\mathrm{H})} & =\sum_{j} \frac{m\left(X_{j}\right)}{m(H)} 10^{\varepsilon_{\text {dust }}\left(X_{j}\right)} \\
& =\sum_{j} \frac{m\left(X_{j}\right)}{m(H)}\left(10^{\varepsilon_{\text {ISM }}\left(X_{j}\right)}-10^{\varepsilon_{\text {gas }}\left(X_{j}\right)}\right),
\end{aligned}
$$

where $m(\mathrm{X})$ is the atomic mass of element $\mathrm{X}$ and $\varepsilon_{\text {dust }}(X), \varepsilon_{I S M}(X)$, and $\varepsilon_{\text {gas }}(X)$ are the logarithmic solid-phase, total ISM, and measured gas-phase abundances of element $X$. We use a combination of measurements and estimates based on MW depletions from Jenkins [3] for $\varepsilon\left(X_{j}\right)$. The GDRs that we compute by scaling our observed amounts of $\mathrm{Fe}$ and $\mathrm{Si}$ by this value are shown in Figure 2.

\section{Discussion}

The depletion range of $\mathrm{Zn}$ and $\mathrm{P}$ is, within the uncertainties, the same in the LMC, SMC, and MW. These elements are completely in the gas-phase along MW sightlines with low overall depletions and could not physically be less depleted. In the LMC, the Si depletion range is consistent with being equal to the MW range but $\mathrm{Cr}$ and Fe are systematically less depleted. In the SMC, Si, $\mathrm{Cr}$, and Fe are systematically less depleted than in the MW or LMC. The SMC depletion ranges for $\mathrm{Si}$ and $\mathrm{Cr}$ are particularly noteworthy, since they are consistent with there being no $\mathrm{Si}$ or $\mathrm{Cr}$ in dust at the lowest overall depletion values.

Our GDR values apply on small size scales. To quantitatively compare them to GDRs from other studies, which are usually based on emission measurements from regions with diameter greater than a parsec, we would need to estimate the effects of averaging over large regions. We would also need to make assumptions about the value of the GDR in dense molecular clouds, where it may further decrease due to grain growth by mantling and accretion, processes our depletion measurements are insensitive to. Qualitatively, our GDRs (250-1200 in the LMC, 700-10000 in the SMC) agree with the values in [11] and disagree with the values in Leroy et al. [10] (150-250 in the LMC, 150-700 in the SMC).

\section{Conclusions}

We have used new and archival UV absorption spectroscopy to find that:

- The depletion ranges of Zn and P are similar in the LMC, SMC, and MW.

- $\mathrm{Si}, \mathrm{Cr}$, and Fe become progressively less depleted from the MW to the LMC to the SMC. 
- The mass GDRs of the LMC and SMC range between 250-1200 and 700-10000, respectively.

\section{References}

[1] A. Li and B. T. Draine, Infrared Emission from Interstellar Dust. II. The Diffuse Interstellar Medium, ApJ, 554, 2778 (2001).

[2] B. D. Savage and K. R. Sembach, Interstellar Abundances from Absorption-Line Observations with the Hubble Space Telescope, Annu. Rev. Astro. Astrophys., 34279 (1996).

[3] E. B. Jenkins, A unified representation of gas-phase element depletions in the interstellar medium, ApJ, 700, 21299 (2009).

[4] A. M. Wolfe, et al., Damped Ly $\alpha$ Systems, Annu. Rev. Astro. Astrophys., 43, 1861 (2005).

[5] A. Rémy-Ruyer et al., Gas-to-Dust mass ratios in local galaxies over a 2 dex metallicity range, arXiv (2013), $1312.3442 \mathrm{v} 1$.

[6] K. D. Gordon et al., A Quantitative Comparison of the Small Magellanic Cloud, Large Magellanic Cloud, and Milky Way Ultraviolet to Near-Infrared Extinction Curves, ApJ, 594, 1279 (2003).

[7] L. A. Valencic, et al., Ultraviolet Extinction Properties in the Milky Way, ApJ, 616, 2912 (2004).

[8] F. Galliano et al., ISM properties in low-metallicity environments. III. The spectral energy distributions of II Zw 40, He 2-10 and NGC 1140, A\&A, 434, 3867 (2005).

[9] T. P. Snow and B. J. McCall, Diffuse Atomic and Molecular Clouds, Annu. Rev. Astro. Astrophys., 44, 1367 (2006).

[10] A. K. Leroy et al., The CO-to-H2 Conversion Factor from Infrared Dust Emission across the Local Group, ApJ, 737, 112 (2011).

[11] J. Roman-Duval, et al., Dust and gas in the Magellanic Clouds from the HERITAGE Herschel Key Project. II. Variations of the apparent gas-to-dust ratio with surface density and across ISM phases (submitted).

[12] S. Osterman et al., The Cosmic Origins Spectrograph: on-orbit instrument performance, Astrophysics and Space Science, 335257 (2011).

[13] W. P. Blair et al., The Far Ultraviolet Spectroscopic Explorer Legacy in the Magellanic Clouds: An Online Stellar Sight Line Atlas, Publications of the Astronomical Society of the Pacific, 121634 (2009).

[14] P. J. Green, Reversible jump Markov chain Monte Carlo computation and Bayesian model determination, Biometrika, 82, 4711 (1995).

[15] D. E. Welty, et al., Interstellar $\mathrm{H}$ I and $\mathrm{H}_{2}$ in the Magellanic Clouds: an expanded sample based on ultraviolet absorption-line data, ApJ, 745, 2173 (2012).

[16] G. J. Ferland et al., The 2013 Release of Cloudy, Revista Mexicana de Astronomía y Astrofísica Vol. 49, 49137 (2013).

[17] N. Grevesse, et al., The chemical composition of the Sun, Astrophysics and Space Science, 328, 1179 (2010).

[18] F. A. Chekhonadskikh, Abundances and absolute stellar magnitudes for $F$ and $G$ supergiants of Magellanic Clouds, Kinemat. Phys. Celest. Bodies, 28, 3128 (2012). 